\title{
Energy Efficiency Analysis of Next-Generation Passive Optical Network (NG-PON) Technologies in a Major City Network
}

\author{
Sofie Lambert ${ }^{1}$, Julio Montalvo ${ }^{2}$, José A. Torrijos ${ }^{2}$, Bart Lannoo $^{1}$, Didier Colle ${ }^{1}$, Mario Pickavet ${ }^{1}$ \\ ${ }^{1}$ Ghent University - iMinds, Gaston Crommenlaan 8 (B 201), 9050 Gent, Belgium \\ Tel: +32 933 14977, Fax: +32933 14899, e-mail: sofie.lambert@intec.ugent.be \\ ${ }^{2}$ Telefónica I+D, D. Ramón de la Cruz, 82-8, 28006 Madrid, Spain
}

\begin{abstract}
Ever-increasing bandwidth demands associated with mobile backhaul, content-rich services and the convergence of residential and business access will drive the need for next-generation passive optical networks (NG-PONs) in the long term. At the same time, there is a growing interest in reducing the energy consumption and the associated cost of the access network. In this paper, we consider a deployment scenario in a major city to assess the energy efficiency of various PON solutions from a telecom operator's perspective. We compare five nextgeneration technologies to a baseline GPON deployment offering similar bandwidths and Quality of Service (QoS) for best-effort high speed connectivity services. We follow two approaches: first, we consider a fixed split ratio (1:64) in an existing Optical Distribution Network (ODN); next, we consider an upgraded ODN with an optimized split ratio for the specific bandwidth and QoS values.

For medium bandwidth demands, our results show that legacy PONs can be upgraded to 10G PON without any ODN modification. For future applications that may require access rates up to $1 \mathrm{~Gb} / \mathrm{s}$, NG-PON2 technologies with higher split ratios and increased reach become more interesting systems, offering the potential for both increased energy efficiency and node consolidation.
\end{abstract}

Keywords: energy consumption, next-generation passive optical networks, split ratio

\section{INTRODUCTION}

Fiber-based gigabit passive optical networks (G/E PONs) are currently being deployed by operators in several countries, offering much higher bandwidths than traditional copper-based access networks. Deployments of $10 \mathrm{~Gb} / \mathrm{s}$ capable PONs (XG-PON1) are expected in the next couple of years. In the long term, exponential bandwidth demand growth due to the rise of new video services (such as ultra High Definition and 3D formats) in all flavors ( $\mathrm{VoD}$, Peer to Peer) and the potential convergence of wireless and wireline access will necessitate the deployment of even faster next-generation PONs beyond $10 \mathrm{~Gb} / \mathrm{s}$, referred to as NG-PON2s [1].

Due to rising energy prices and the growing awareness of climate change, energy efficiency will be an important factor to take into consideration when analyzing the operational expenditures and carbon footprint of the different NG-PON2 technologies [1]. In this paper, we assess the energy efficiency of G/E PON, 10G PON and four NG-PON2 candidates. Our analysis focuses on the power consumption at the Central Offices (COs) of the network provider. For a general analysis of power saving strategies in home networks and in a PON Optical Network Unit (ONU), we refer to [2], where energy efficiency improvement options in hardware design and virtualization are discussed.

In this work, we analyze how energy efficiency at the $\mathrm{CO}$ is affected by the chosen network deployment and by user demand. We consider a deployment scenario in a major European city to get a more realistic estimation than a purely component-based analysis, taking into account the implications of technology-dependent physical reach over a target area with a limited number of COs.

The paper is organized as follows. In the next section, we introduce our model for the access network and its power consumption, along with the various PON solutions under study. In section 3, we introduce the topology of the city deployment and our model for user demand. Next, we explain how quality of service (QoS) is quantified and how the network is dimensioned (i.e. how we determine the split ratio and uplink capacity) in section 4 . Our results are presented in section 5, followed by the main conclusions in section 6 .

\section{ACCESS NETWORK AND POWER CONSUMPTION MODEL}

In this section, we present our model for the access network and the components of its power consumption. Next, we introduce the various PON technologies which will be considered in this paper, with their specific power consumption parameters and physical constraints.

\subsection{Power consumption model}

Fig. 1 gives a schematic overview of our power consumption model for the optical access network. Power consumption at the customer side is not included in our results, but can be derived from the ONU values in Table 1. ONU power consumption is constant and independent of the chosen deployment scenario. Note that in order to include DC/DC conversion losses, the ONU values need to be multiplied by a factor 1.25 [3]. 


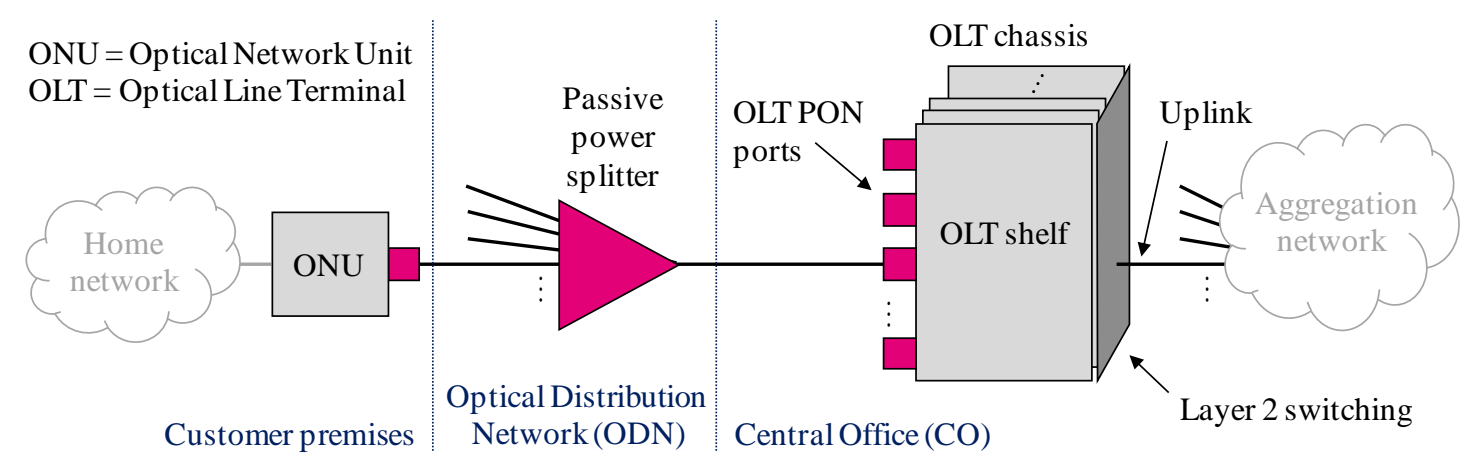

Figure 1. Schematic overview of the access network and its power consumption components. Power consumption values for the ONU and for the OLT PON ports are given in Table 1. Power consumption values for Layer 2 switching and Uplink ports are given in section 2.1. We assume a fully passive ODN.

The access network power consumption at the $\mathrm{CO}$ is calculated as the sum of three contributions:

- $\quad$ OLT PON ports: \#OLT ports x power consumption per port (given in Table 1);

- $\quad$ Layer 2 switching: \#OLT chassis x PONs/chassis x bandwidth (DS+US) x 1 W/Gbps [4];

- Uplink ports: \#OLT chassis $\mathrm{x}$ uplink energy consumption. The uplink energy consumption depends on the required uplink capacity and is obtained by combining bidirectional uplink ports with capacities 1, 10, 40, 100, 400 and $1000 \mathrm{~Gb} / \mathrm{s}$ which consume 7, 38, 105, 205, 560 and $1100 \mathrm{~W}$ respectively [5].

The equipment count (number of OLT ports \& OLT chassis) and required uplink capacity are calculated based on the user demand and QoS scenario, as described in sections 3 and 4. For equipment installed at the operator's CO, two overhead factors are taken into account: we multiply the results by a factor of 1.25 to include DC/DC conversion losses and by a site factor of 1.70 to account for the energy consumption of auxiliary equipment such as AC/DC rectifiers, ventilation and air conditioning, auxiliary power units and batteries [3]. The total power consumption of the deployment is divided by the number of connected users to obtain the power consumption per user. Note that energy saving mechanisms such as sleep modes are not considered in this work.

\subsection{Technologies considered}

A number of relevant candidates for NG-PON2 systems have been recognized by the FSAN (Full Services Access Network) group [1]. In this work, we focus on the following options: 40G (XLG) PON, Time-shared Wavelength Division Multiplexing (TWDM) PON, Orthogonal Frequency Division Multiplexing (OFDM) PON and Coherent Dense WDM (Co-DWDM) PON. We will compare the energy efficiency of these solutions with that of two previous PON generations: Gigabit/Ethernet PON (GPON/EPON) with B+ optics and 10G PON (XG-PON1 E2 class). An overview of the main characteristics of the considered PON solutions is given in Table 1. We have selected the maximum budget class for each technology. Only passive long-reach approaches are considered, avoiding the use of active elements in the Optical Distribution Network (ODN). The deployment is power splitter-based (as opposed to wavelength splitter-based) to ensure compatibility with legacy ODNs. In case of wavelength division multiplexing, the wavelength-selective components are installed at the customer premises and at the $\mathrm{CO}$.

In Table 1, it can be seen that the power consumption per OLT PON port increases when the PON bandwidth increases. For OFDM PON and Co-DWDM PON, the power consumption depends on the number of users that can be served by the OLT port. To serve more users, more digital signal processing and (in some cases) additional transmitters and receivers are required.

Table 1. PON technologies: performance and power consumption parameters. Power consumption parameters are based on [3], assuming the same ONU power consumption for Co-DWDM PON as for XLG PON and scaling power for OLT processing \& amplifier with bandwidth and the number of users.

\begin{tabular}{|c|c|c|c|c|c|c|}
\hline Technology & $\begin{array}{c}\text { Max. } \\
\text { budget }(\mathrm{dB})\end{array}$ & $\begin{array}{l}\text { Attenuation } \\
(\mathrm{dB} / \mathrm{km})\end{array}$ & $\begin{array}{c}\text { Bandwidth / PON } \\
\text { DS/US (Gb/s) }\end{array}$ & $\begin{array}{l}\text { PONs / } \\
\text { chassis }\end{array}$ & $\begin{array}{c}\text { power / OLT } \\
\text { port }(\mathrm{W})\end{array}$ & $\begin{array}{c}\text { power / } \\
\text { ONU (W) }\end{array}$ \\
\hline G/E PON (B+) & 28 & 0.6 & $2.5 / 1.25$ & 128 & 2 & 5.1 \\
\hline 10G PON (E2) & 35 & 0.6 & $10 / 2.5$ & 64 & 5 & 7.1 \\
\hline XLG PON & 31 & 0.6 & $40 / 10$ & 32 & 17 & 14.6 \\
\hline TWDM PON & 38.5 & 0.4 & $4 \times 10 / 4 \times 2.5$ & 32 & 20 & 7.1 \\
\hline OFDM PON & 34.5 & 0.6 & $40 / 10$ & 32 & $3.5+0.5 \mathrm{xN}_{\text {users }} *$ & 11.1 \\
\hline Co-DWDM PON & 43 & 0.4 & $1.25 / 1.25 \times \mathrm{N}_{\text {users }} *$ & 32 & $6+1.2 \mathrm{xN}_{\mathrm{users}} *$ & 14.6 \\
\hline
\end{tabular}

$* \mathrm{~N}_{\text {users }}$ is the maximum number of connected users per OLT PON port (scales with the split ratio) 
Power consumption of the ONU also scales with PON capacity, since faster transceivers consume more power. In case of TWDM PON, four virtual PONs on different wavelengths each deliver $10 \mathrm{~Gb} / \mathrm{s}$ downstream and $2.5 \mathrm{~Gb} / \mathrm{s}$ upstream, therefore we assume the power consumption for a $10 \mathrm{G}$ PON ONU is the same as for a TWDM PON ONU (which is tuned to a single wavelength). In the considered model, the power consumption for an OFDM PON ONU is slightly lower than for an XLG PON ONU because, in the latter, more functions are performed at the 40G line rate (de-scrambling, Forward Error Correction decoding, dispersion compensation...), while the same functions can either be performed at lower rates or are not required in the OFDM PON ONU.

\section{SCENARIO DESCRIPTION}

In this section, the city deployment algorithm is described, covering the city topology and the methodology for determining the equipment inventory. Next, we introduce the user behavior model and the dynamic bandwidth allocation principle.

\subsection{City Deployment Tool}

The city deployment topology is obtained from earlier research in the ACCORDANCE FP7 project [6]. An area of $70 \mathrm{~km}$ radius around a major European city was analyzed and the results were used to create the reference model, consisting of a central zone surrounded by several smaller, less populated towns and a suburban zone.

The fiber outside plant deployment algorithm requires the following input parameters: percentage of real estate units passed (assumed 60\%), percentage of those real estate units connected (assumed 50\%), PON solution adopted (with technology-dependent max. budget, attenuation and number of PONs/chassis) and desired split ratio. Based on these input parameters, a link budget calculation is performed to obtain the maximum range of the solution under study. XG-PON1 and NG-PON2 technologies require a coexistence WDM filter at the Central Office, for which $1 \mathrm{~dB}$ loss is taken into account. Additional insertion losses are introduced in the splitters: higher split ratios induce more losses.

Every population kernel within the study is supposed to have a $\mathrm{CO}$ close to its geographical centre. Based on the maximum range, the deployment algorithm consolidates these COs as close as possible to the central zone. After the consolidation process, the number of OLT ports and OLT chassis required for the deployment are calculated. The higher the reach of the adopted technology, the lower the number of OLTs and COs in service required, thus the potential for energy efficiency of Long Reach PONs is exploited. The number of OLT ports and OLT chassis is used as input for the power consumption calculation.

\subsection{Service Model, User Demand Model and Dynamic Bandwidth Allocation}

We consider a best-effort internet access service dominating the traffic in the PONs, where a maximum bandwidth, namely $B_{\text {target }}$, is offered to each customer with a minimum percentage of time of availability, namely $p_{\text {avail,min }}$, and offering the same priority to each customer. We only model downstream bandwidth, assuming the upstream bandwidth scales with downstream bandwidth following the technology-dependent PON symmetry ratio $(100 \%, 50 \%$ or $25 \%$, see DS/US bandwidth/PON in Table 1). We adopt the user behavior model from [7], where each user has the same probability $p_{a c t}$ to be active. We extend this model by assuming that users request a fixed target bandwidth $B_{\text {target }}$ when they are active.

For the Time Division Multiple Access (TDMA) technologies, we assume perfect dynamic bandwidth allocation (i.e. without packet loss in the ODN) in both upstream and downstream direction. When $k$ active users from $N$ total independent users are demanding or delivering traffic from/to an OLT interface, the maximum bandwidth that can be offered to each user is

$$
B_{\text {max }}=\frac{\text { PON bandwidth }}{k}, \quad 1 \leq k \leq N
$$

As NG-PON2 protocols are still not defined, only raw bit capacity has been quantified without considering protocol efficiency. When $B_{\max }$ is greater than $B_{\text {target }}$, all active users get the requested $B_{\text {target }}$. However, when there are too many active users, $B_{\max }$ may be smaller than $B_{\text {target }}$, and the active users can only get $B_{\max }$. The probability $p_{\text {avail }}$ that $B_{\text {target }}$ is available equals the probability that the number of active users $k$ is smaller than or equal to $k_{\max }$, given by the cumulative binomial probability [7]

$$
p_{\text {avail }}=\sum_{k=0}^{k_{\max }} \frac{N !}{(N-k) ! k !}\left(1-p_{\text {act }}\right)^{N-k} p_{\text {act }}^{k}, \quad k_{\text {max }}=\left\lfloor\frac{\text { PON bandwidth }}{B_{\text {target }}}\right\rfloor
$$

\section{NETWORK DIMENSIONING}

In order to make a fair energy efficiency comparison between the different PON solutions, we fix the user demand parameters $\left(p_{a c t}\right.$ and $B_{\text {target }}$ ) and the QoS requirements before dimensioning the network. QoS is quantified using two parameters, which are introduced in the following subsection. In subsection 4.2, we describe how the split ratio and uplink capacity are determined. 


\subsection{Quality of Service (QoS) Quantification}

Quality of Service is quantified by two parameters: $p_{\text {avail,min }}(\%)$, the minimum percentage of time that the target bandwidth should be available for each connected user; and MPL (Maximum Packet Loss), the maximum ratio of packets discarded over packets offered in the uplink interface of an OLT chassis (from the OLT to the aggregation network). In our analysis we focus on a best-effort internet service, with moderate Quality of Service requirements: MPL is fixed at $10^{-3}$, and $p_{\text {avail, } \min }=20 \%$.

\subsection{Dimensioning Methodology}

Once the user demand and QoS values are fixed, we use two different approaches to compare the technologies.

In the first approach, we look at the performance of the solutions without modifications of the ODN, considering a fixed legacy split ratio of 1:64 (64 homes passed per PON). For each solution, we calculate $p_{\text {avail }}$ and check if the QoS requirement can be satisfied.

In the second approach, we determine the maximal split ratio at which $p_{\text {avail,min }}$ can be guaranteed for each technology (note that this split ratio may be higher or lower than the legacy value of 1:64, depending on the user demand, the offered bandwidth and the PON technology). There is a trade-off between availability (QoS) and power consumption: increasing the split ratio will decrease availability, but it will also decrease power consumption as the OLT equipment is shared by more users. We assume a maximum split ratio of 1:256, with additional limitations posed by the maximum power budget (listed in Table 1).

In both approaches, the chosen split ratio is then used as input for the city deployment algorithm which calculates the equipment inventory (as described in section 3.1), which in turn is used as input for the power consumption calculation. The split ratio is also used as input for the chassis uplink capacity dimensioning. Based on the user demand, the PON bandwidth (we only consider DS bandwidth as it is always $\geq$ US bandwidth and uplink capacity is symmetrical), the maximum number of connected users per PON and the number of PONs per chassis, we estimate the distribution of the uplink traffic load through simulations. The uplink capacity required to meet the MPL requirement is then calculated using the flow level approach from [8].

\section{RESULTS AND DISCUSSION}

We use the results of the network dimensioning as input for the power consumption calculation described in section 2.1, to obtain values for the power consumption at the $\mathrm{CO}$ for the different PON solutions and for various user demands. We consider both fixed and varying split ratios. The results are discussed below.

\subsection{Fixed split ratio}

In our first analysis approach, we consider a fixed split ratio of 1:64, allowing operators to re-use the legacy ODN of (X)G-PON solutions without modifications. Fig. 2 shows the availability and power consumption of the various solutions for a low user activity $\left(p_{a c t}=0.1\right)$. Increasing the target bandwidth from $100 \mathrm{Mb} / \mathrm{s}$ to $1 \mathrm{~Gb} / \mathrm{s}$ results in a lower availability for G/E PON technologies, but it remains above the threshold of $20 \%$. The power
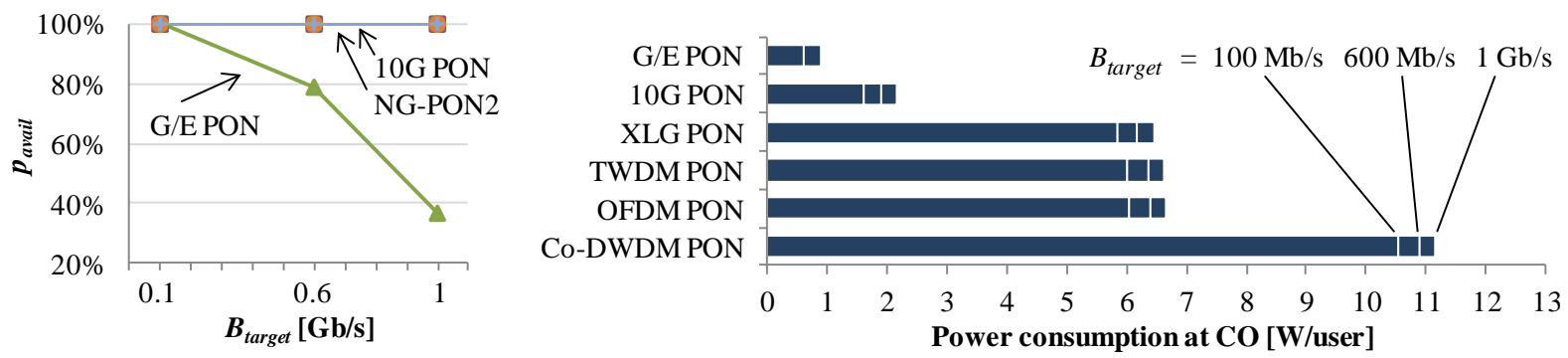

Figure 2. Availability (left) and power consumption (right) of the PON solutions for varying target bandwidths in case of low user activity $\left(p_{a c t}=0.1\right)$, using an existing ODN with split ratio 1:64.
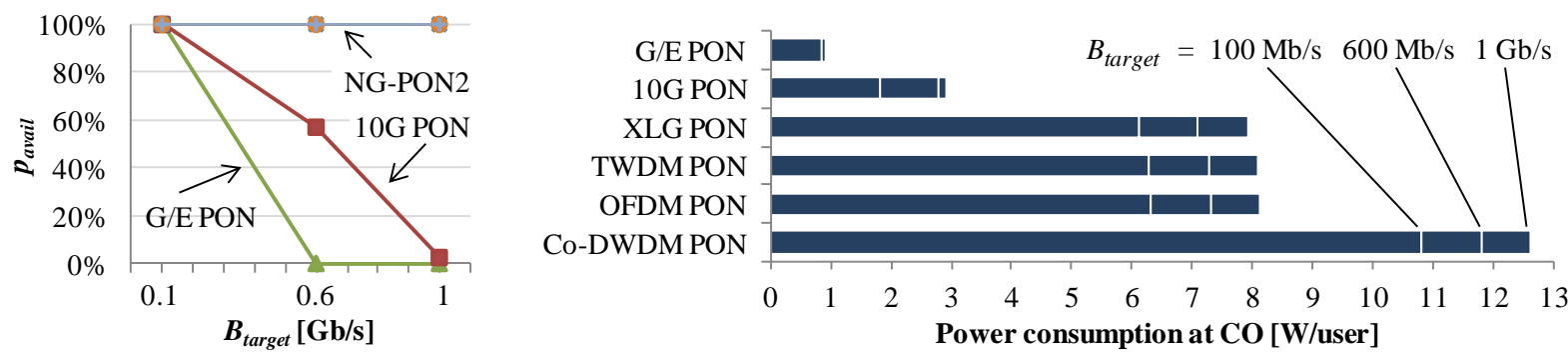

Figure 3. Availability (left) and power consumption (right) of the PON solutions for varying target bandwidths in case of high user activity $\left(p_{a c t}=0.5\right)$ using an existing ODN with split ratio 1:64. 

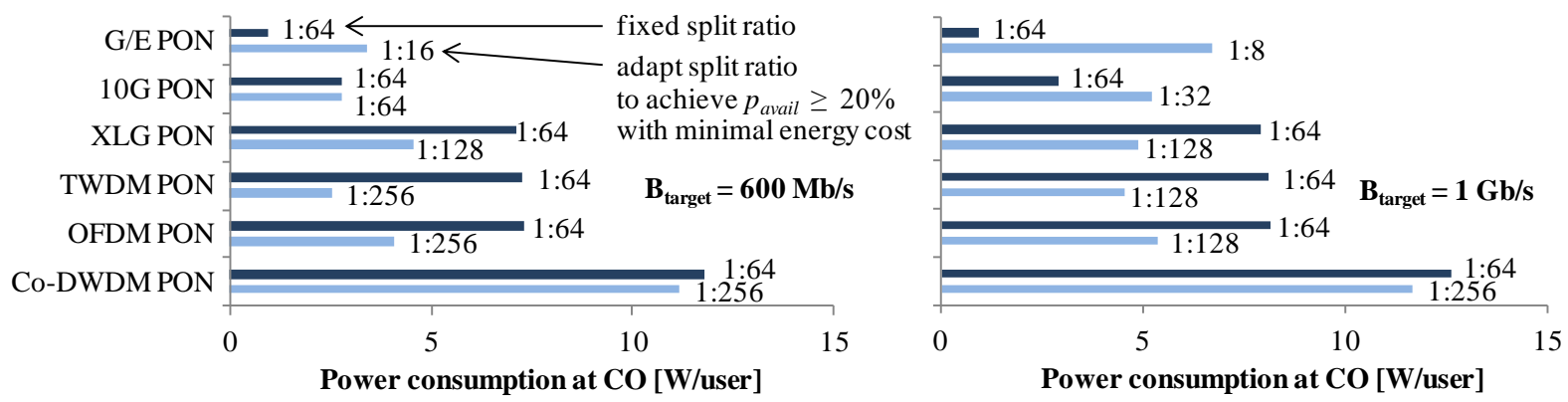

Figure 4. Power consumption of the PON solutions for target bandwidth $600 \mathrm{Mb} / \mathrm{s}$ (left) and $1 \mathrm{~Gb} / \mathrm{s}$ (right) in case of high user activity $\left(p_{a c t}=0.5\right)$ : results for an existing ODN with split ratio 1:64 (dark bars) versus a flexible ODN (light bars). The chosen split ratios are indicated next to the bars.

consumption is not impacted significantly by a change in $B_{\text {target }}$, and is lowest for the G/E PON solutions. So in this case, even the legacy technologies can provide high speed connectivity services with acceptable QoS and with lower power consumption than XG-PON and NG-PON2 technologies.

When considering a higher user activity, as in Fig. $3\left(p_{a c t}=0.5\right)$, availability becomes unacceptably low for $\mathrm{G} / \mathrm{E}$ PON at a target bandwidth of $600 \mathrm{Mb} / \mathrm{s}$. A switchover to XG-PON could improve QoS greatly at a relatively low energy cost. If bandwidth requirements grow even further, up to $1 \mathrm{~Gb} / \mathrm{s}$, NG-PON2 technologies will be needed. The expense of providing these high speed services in a legacy ODN using NG-PON2 technologies is a high increase in the energy demand.

\subsection{Comparison with a flexible split ratio}

In our second analysis approach, we consider some flexibility in the ODN: splitters can be modified to adapt the split ratio, and COs can be consolidated by eliminating active nodes. For each technology, we select the highest split ratio at which the availability $p_{\text {avail }}$ is still above $20 \%$. By increasing the split ratio, we can fully take advantage of the higher capacities of next-generation PON solutions. This could make them a more attractive option in case of high user demands. Therefore, we consider the high user activity scenario here $\left(p_{a c t}=0.5\right)$.

Fig. 4 shows the power consumption of a fixed versus a flexible split ratio scenario, for a target bandwidth of $600 \mathrm{Mb} / \mathrm{s}$ (left) and $1 \mathrm{~Gb} / \mathrm{s}$ (right). For a medium target bandwidth of $600 \mathrm{Mb} / \mathrm{s}$, the power consumption per user for NG-PON2 solutions can be decreased significantly by deploying higher split ratios. This would allow for CO consolidation and thus easier network management, while offering similar QoS at a comparable energy cost.

In case of high target bandwidths of $1 \mathrm{~Gb} / \mathrm{s}$ (right), the quality of service for G/E PON and 10G PON becomes unacceptable when considering a fixed split ratio, as was shown in the previous section. These technologies would need to be deployed at lower split ratios in order to reach the required QoS. In this case, NG-PON2 technologies become a more interesting option, as they are more energy efficient, in addition to offering the potential for $\mathrm{CO}$ consolidation thanks to their higher split ratios.

\subsection{Performance of the different NG-PON2 solutions}

From our results, it is clear that Co-DWDM PON has a higher power consumption per user at the CO in every scenario; however, it must be noted that this solution offers the advantage of $100 \%$ bandwidth availability and lowest traffic latency, which may be useful for specific applications such as business services or mobile backhauling. The power consumption values for the other technologies are close to each other; in this case, power consumption in the ONU could be a differentiating factor, with TWDM requiring less energy than OFDM and XLG PON. However, the implementation of power saving strategies such as sleep modes may still change the relative proportions between these three solutions.

\section{CONCLUSIONS}

We have studied the energy efficiency of various PON technologies on the operator side for best-effort highspeed connectivity services up to $1 \mathrm{~Gb} / \mathrm{s}$, by considering a deployment scenario in a major city. Our results show that for high user activity, legacy PON technologies can provide access speeds up to $100 \mathrm{Mb} / \mathrm{s}$ with the required QoS using presently deployed networks with a split ratio of 1:64. To a certain extent, increasing demands up to $600 \mathrm{Mb} / \mathrm{s}$ can be met by using 10G PON without changes to the ODN.

As server capacities increase and cache systems get information closer to the OLTs, as well as in case of specific applications such as business services and mobile backhaul, access rates up to $1 \mathrm{~Gb} / \mathrm{s}$ may be required in the future. For these high user demands it's more interesting to move to NG-PON2 technologies with higher split ratios and increased reach, which offer the potential for $\mathrm{CO}$ consolidation (simplifying network management) in addition to enhanced energy efficiency. 


\section{ACKNOWLEDGEMENTS}

This research has received funding from the EU FP7 projects TREND (ICT-257740) and DISCUS (ICT318137). The first author is funded by an IWT-Vlaanderen grant.

\section{REFERENCES}

[1] P. Chanclou, et al:: Network operator requirements for the next generation of optical access networks, IEEE Network, vol. 26. pp. 8-14, March/April 2012.

[2] J. Montalvo, et al:: Energy efficiency in PON home network scenarios with network enhanced residential gateways, in Proc. IEEE ICNSC 2013, Evry, France, April 2013, paper ThB03.2.

[3] B. Skubic, et al.: Energy-efficient next-generation optical access networks, IEEE Communications Magazine, vol. 50. pp. 122-127, Jan. 2012.

[4] OASE FP7 Deliverable 8.5: Integrated OASE results overview, Mar. 2013. Available online at http://www.ict-oase.eu/index.php?page=120\&

[5] W. Van Heddeghem, et al:: Power consumption modeling in optical multilayer networks, Photonic Network Communications, vol. 24. pp. 86-102, Oct. 2012

[6] Accordance project, http://www.ict-accordance.eu/

[7] J. Segarra, V. Sales, J. Prat: Access services availability and traffic forecast in PON deployment, in Proc. ICTON 2011, Stockholm, Sweden, June 2011, paper Tu.A6.4.

[8] W. Lautenschlaeger, W. Frohberg: Bandwidth dimensioning in packet-based aggregation networks, in Proc. Int. Telecommunications Network Strategy and Planning Symp., Budapest, Hungary, Oct. 2008. 